\title{
FINITE TYPE LINK INVARIANTS AND THE NON-INVERTIBILITY OF LINKS
}

\author{
XIAO-SONG LiN
}

\begin{abstract}
We show that a variation of Milnor's $\bar{\mu}$-invariants, the so-called Campbell-Hausdorff invariants introduced recently by Stefan Papadima, are of finite type with respect to marked singular links. These link invariants are stronger than quantum invariants in the sense that they detect easily the non-invertibility of links with more than one components. It is still open whether some effectively computable knot invariants, e.g. finite type knot invariants (Vassiliev invariants), could detect the non-invertibility of knots.
\end{abstract}

\section{Introduction}

It is well-known that the indeterminacy of Milnor's $\bar{\mu}$-invariants [?, ?] is inadequate for various link classification problems (see, for example, $[?, ?])$. Moreover, the indeterminacy of $\bar{\mu}$-invariants depends on individual links so that a fixed $\bar{\mu}$-invariant takes values in different cyclic groups on different links. This awkwardness is the reason why in [?, ?], where one studies whether $\bar{\mu}$-invariants are of finite type, one has to drop off the indeterminacy at all to consider only string links.

Recently, based on the work of M. Markl and S. Papadima [?] on an algebraic parameterization for the set of (Mal'cev completed) fundamental groups of the spaces with fixed first two Betti numbers (a moduli space of $h_{1,2}$-marked groups), S. Papadima introduced a new class of link concordance invariants [?]. For links with fixed number of components, these socalled Campbell-Hausdorff invariants take values in a fixed moduli space. These invariants can be thought of as refinements of $\bar{\mu}$-invariants in that the latter are derived using the Magnus expansion of free groups while the former using the Campbell-Hausdorff expansion (and hence the name).

The moduli space of $h_{1,2}$-marked groups is a tower of orbit spaces of vector spaces of derivations of certain free nilpotent Lie algebras under the action of a prounipotent group. To obtain the Campbell-Hausdorff invariants, one applies the Campbell-Hausdorff expansion to the quotients of the fundamental group of a link complement by its lower central series. The

Received February 1, 1996.

Research supported in part by NSF. 
indeterminacy is thus reflected in the action of that prounipotent group. In [?], we have argued that, regardless of the indeterminacy, link concordance invariants obtained from any expansion of free groups are of finite type. The difficult now is that the moduli space where the Campbell-Hausdorff invariants live is not a vector space, although some linear structures do survive there. To overcome this difficulty, we introduce the notion of marked singular links, which are the usual singular links with a mark on each component away from double points. Under isotopy of marked singular links, the marks are only allow to move without passing through double points. Of course, isotopy classes of marked links are in one-one correspondence with isotopy classes of unmarked links. The Campbell-Hausdorff link invariants can be used to derive invariants of marked singular links. For a fixed Campbell-Hausdorff invariant, we show that the derived marked singular link invariant vanishes when the number of double points is large enough. In that sense, we say that the Campbell-Hausdorff invariants are of finite type with respect to marked singular links. It will certainly be interesting to explore systematically the combinatorics of link invariants of finite type with respect to marked singular links.

Being of finite type (with respect to unmarked singular links) is a property of link invariants obeyed by the coefficients of appropriate power series expansions of all link invariants derived from finite dimensional representations of simple Lie algebras through the theory of quantum groups (generalizations of the Jones polynomial) [?, ?, ?]. Since then, there has been a question about whether (linear combinations of) those finite type link invariants derived from quantum groups (quantum invariants) are the only finite type link invariants. Notice that quantum invariants have an additional property that their values will not change when the orientation of each component of a given link is reversed due to a certain kind of self-duality of finite dimensional representations of simple Lie algebras. So one was asked whether finite type link invariants can detect the noninvertibility of a link. Recently, P. Vogel was able to answer the former question in negative through a very complicated combinatorial manipulation of chord diagrams. On the other hand, it is very simple to come up with examples of Campbell-Hausdorff invariants which detect the noninvertibility of a link with more than one components. Therefore, such a Campbell-Hausdorff invariant can not be determined by quantum invariants on links with more than one components. It is still not known whether finite type knot invariants can detect the non-invertibility of knots (we conjectured that the answer is no several years ago).

Notice that the Campbell-Hausdorff invariants are derived directly from the fundamental groups of links complements, while it has been a question whether the quantum link invariants have anything to do with the fun- 
damental groups of link complements. We have shown here some of the similarities and differences of the Campbell-Hausdorff invariants and the quantum invariants. This, we think, is of some significance.

We will review the background in Section 2. The statement of the main result and its proof are in Section 3. In Section 4, we include a proof of the well-known fact that quantum invariants can not detect the noninvertibility of a link. Section 5 contains an example and some comments.

We are very grateful to Dror Bar-Natan for a critical reading of the original version of the paper. His comments helped us to clear up some confusions in our understanding of the works of Markl-Papadima and Papadima.

\section{Background}

2.1. The works of Markl-Papadima and Papadima. We first summarize briefly the works of Markl-Papadima [?] and Papadima [?]. See also [?].

Let $X$ and $Y$ be the $\mathbb{Q}$-vector spaces spanned by $\left\{x_{1}, \ldots, x_{n}\right\}$ and $\left\{y_{1}, \ldots, y_{n}\right\}$, respectively. Let $\mathbb{L}_{*}^{*}=\mathbb{L}(X \oplus Y)$ be the free bigraded $\mathbb{Q}$-Lie algebra generated by $X \oplus Y$, where the upper degree is given by the bracket length and the lower degree comes from setting $|X|=0$ and $|Y|=1$. Let $\hat{\mathbb{L}}=\hat{\mathbb{L}}_{*}$ be the completion of $\mathbb{L}_{*}^{*}$ with respect to the upper degree, i.e. with respect to the filtration $F_{k} \mathbb{L}_{*}=\mathbb{L}_{*}^{\geq k}$. We have an induced filtration $F_{k} \hat{\mathbb{L}}$ for $\hat{\mathbb{L}}$.

Consider $\hat{M}=\operatorname{Der}_{-1}^{+} \hat{\mathbb{L}}$, the vector space of continuous derivations $\partial$ of $\hat{\mathbb{L}}$, which are homogeneous of lower degree -1 and have the property that $\partial F_{k} \hat{\mathbb{L}} \subset F_{k+1} \hat{\mathbb{L}}, \forall k$, filtrated by

$$
F_{s} \hat{M}=\left\{\partial \mid \partial F_{k} \hat{\mathbb{L}} \subset F_{k+s} \hat{\mathbb{L}}, \forall k\right\} .
$$

Since $\hat{\mathbb{L}}$ is free, a derivation $\partial \in \operatorname{Der}_{-1}^{+} \hat{\mathbb{L}}$ is completely determined by its restriction to the free generators. Together with the assumption that $\partial$ is of lower degree -1 and $\partial F_{k} \hat{\mathbb{L}} \subset F_{k+1} \hat{\mathbb{L}}, \forall k$, we may identify $\partial$ with a series

$$
\left.\partial=\partial_{1}+\partial_{2}+\cdots+\partial_{s}+\cdots, \quad \partial_{s} \in \operatorname{Hom}\left(Y, \mathbb{L}_{X}^{s+1}\right)\right), \forall s
$$

where $\hat{\mathbb{L}}_{X}$ is the completed free $\mathbb{Q}$-Lie algebra on $X$, grade by the bracket length (upper degree) and filtrated by $F_{s} \hat{\mathbb{L}}_{X}=\hat{\mathbb{L}}_{\bar{X}} \geq s$. We have

$$
\partial \in F_{s} \hat{M} \quad \Longleftrightarrow \quad \partial_{<s}=0,
$$

and obviously,

$$
\hat{M} / F_{s} \hat{M} \cong \operatorname{Hom}\left(Y, \hat{\mathbb{L}}_{\bar{X}}^{\geq 2} / \hat{\mathbb{L}}_{\bar{X}}^{\geq s+1}\right) .
$$


Consider the group $U$ of continuous lower degree zero Lie algebra automorphisms $u$ of $\hat{\mathbb{L}}$ with the property that $\operatorname{gr}^{1} u=$ id (with respect to the filtration $F_{s} \hat{\mathbb{L}}$. It acts on $\hat{M}$ by conjugation and the action preserves the filtration $F_{s} \hat{M}$. Define

$$
\mathcal{M}_{s}=U \backslash \hat{M} / F_{s} \hat{M}
$$

be the double quotient of $\hat{M}$ by $F_{s} \hat{M}$ and the $U$-action. We have a natural projection

$$
\operatorname{pr}_{s}^{s+1}: \mathcal{M}_{s+1} \longrightarrow \mathcal{M}_{s}
$$

Remark. It is very important that the space $\mathcal{M}_{s}$ is the orbit space of the vector space $\hat{M} / F_{s} \hat{M}$ under the action of $U$ and not the quotient vector space. In fact, the latter is trivial. Nevertheless, some linear structures do survive in $\mathcal{M}_{s}$. For example, there is a natural scalar multiplication on $\mathcal{M}_{s}$. For each $s$, there is a unique element $0 \in \mathcal{M}_{s}$ invariant under the scalar multiplication and preserved by the projection $\mathrm{pr}_{s}^{s+1}$. Moreover, $\left(\operatorname{pr}_{s}^{s+1}\right)^{-1}(0) \subset \mathcal{M}_{s+1}$ is a vector space for each $s$. See the discussion at the beginning of Section 5 .

Now consider a classical link $L \in S^{3}$, consisting of $n$ disjointly embedded circles, which are ordered and oriented. Let $G=\pi_{1}\left(S^{3} \backslash L\right)$ be the fundamental group of the link complement of $L$, and $\Gamma_{s} G$ be the lower central series of $G$ with $\Gamma_{1} G=G$ and $\Gamma_{s}=\left(\Gamma_{s-1}, G\right)$, where (, ) stands for the group commutator. The existence of a system of meridians and longitudes $m_{i}, l_{i} \in G(1 \leq i \leq n)$ unique modulo conjugations $g_{i} m_{i} g_{i}^{-1}, g_{i} l_{i} g_{i}^{-1}$ by $g_{i} \in G(1 \leq i \leq n)$ determines a peripheral structure on $G$. The tower of nilpotent groups $G / \Gamma_{s+1} G$ inherits such a peripheral structure. The structured tower of nilpotent groups $G / \Gamma_{s+1} G$ turns out to be an $I$-equivalence invariant of links ([?]). Here two links $L$ and $L^{\prime}$ are $I$-equivalent if there is a proper (not necessarily locally flat) imbeddings of $S^{1} \times I_{1} \amalg \cdots \amalg S^{1} \times I_{n}, I_{i}=I=[0,1]$, into $S^{3} \times I$ such that $L=S^{1} \times\{0\} \coprod \cdots \coprod S^{1} \times\{0\} \subset S^{3} \times\{0\}$ and $L^{\prime}=S^{1} \times\{1\} \coprod \cdots \coprod S^{1} \times\{1\} \subset S^{3} \times\{1\}$. In particular, every knot is $I$-equivalent to the unknot.

The nilpotent group $G / \Gamma_{s+1} G$ admits a very simple presentation. Let $\mathbb{F}=\mathbb{F}\left(x_{1}, \ldots, x_{n}\right)$ be the free group generated by $x_{1}, \ldots, x_{n}$.

Theorem 2.1. (Milnor [?]) Given an n-component link $L$ with the link group $G=\pi_{1}\left(S^{3} \backslash L\right)$. For every $s \geq 1$, there exist elements $l_{i}^{(s)} \in \mathbb{F}$ $(1 \leq i \leq n)$, such that

- $l_{i}^{(s+1)} \equiv l_{i}^{(s)} \bmod \Gamma_{s} \mathbb{F}, 1 \leq i \leq n$;

- $G / \Gamma_{s+1} G$ is isomorphic to

$$
\left\{x_{1} \ldots, x_{n} \mid\left(l_{i}^{(s)}, x_{i}\right), 1 \leq i \leq n, \Gamma_{s+1} \mathbb{F}\right\}
$$


as structured groups where the peripheral structure of the latter group is given by $x_{i}, l_{i}^{(s)}, 1 \leq i \leq n$.

The Campbell-Hausdorff representation is an injective homomorphism

$$
\rho: \mathbb{F}\left(x_{1}, \ldots, x_{n}\right) \longrightarrow \hat{\mathbb{L}}_{X}
$$

into the Campbell-Hausdorff group of $\hat{\mathbb{L}}_{X}$ given by $\rho\left(x_{i}\right)=x_{i}, 1 \leq i \leq n$ and the Campbell-Hausdorff formula

$$
\rho(g h)=\rho(g)+\rho(h)+\frac{1}{2}[\rho(g), \rho(h)]+\cdots, \quad g, h \in \mathbb{F}
$$

where [, ] is the algebraic commutator (see [?] for an explicit form of the Campbell-Hausdorff formula). For an $n$-component link $L$ with the structured group $G$, use the presentation (2.2), one can define a homomorphism $\partial^{(s)} \in \operatorname{Hom}\left(Y, \hat{\mathbb{L}}_{X}^{2}\right)$ by

$$
\partial^{(s)}\left(y_{i}\right)=\left[\rho\left(l_{i}^{(s)}\right), x_{i}\right], \quad 1 \leq i \leq n .
$$

Under the natural identification (2.1), we have a projection

$$
\operatorname{Hom}\left(Y, \hat{\mathbb{L}}_{\bar{X}}^{\geq 2}\right) \longrightarrow \operatorname{Hom}\left(Y, \hat{\mathbb{L}}_{\bar{X}}^{\geq 2} / \hat{\mathbb{L}}_{\bar{X}}^{\geq s+1}\right) \longrightarrow \mathcal{M}_{s} .
$$

Then, define $C H^{(s)}(L) \in \mathcal{M}_{s}$ to be the image of $\partial^{(s)}$ under this projection. We have

Theorem 2.2. (Papadima [?]) $C H^{(s)}(L) \in \mathcal{M}_{s}$ depends only on the $I$ equivalence class of $L$. Moreover, we have

$$
p r_{s}^{s+1}\left(C H^{(s+1)}(L)\right)=C H^{(s)}(L) .
$$

2.2. Finite type link invariants. Here we briefly review the results in [?].

First, we will refer the reader to the standard references [?, ?, ?] for the definition finite type knot invariants (or Vassiliev invariants). The definition can be easily generalized to links and string links [?, ?].

Briefly, an $n$-component string link $\sigma$ is a self-concordance of $n$ fixed points in the plane $\mathbb{R}^{2}$. For each $s$, such a string link $\sigma$ induces an automorphism

$$
\phi_{\sigma}^{(s)}: \mathbb{F} / \Gamma_{s+1} \mathbb{F} \longrightarrow \mathbb{F} / \Gamma_{s+1} \mathbb{F}
$$

given by

$$
\phi_{\sigma}^{(s)}\left(x_{i}\right)=l_{i}^{(s)} x_{i}\left(l_{i}^{(s)}\right)^{-1}, \quad 1 \leq i \leq n
$$

for certain $l_{i}^{(s)} \in \mathbb{F}$ and

$$
l_{i}^{(s+1)} \equiv l_{i}^{(s)} \quad \bmod \quad \Gamma_{s} \mathbb{F} .
$$


Let $\hat{\mathbb{P}}=\hat{\mathbb{P}}\left(X_{1}, \ldots, X_{n}\right)$ be the ring of formal power series (with rational coefficients) in non-commutative variables $X_{1}, \ldots, X_{n}$. It comes naturally with a grading and a filtration $F_{s} \hat{\mathbb{P}}$. An expansion of the free group $\mathbb{F}=$ $\mathbb{F}\left(x_{1}, \ldots, x_{n}\right)$ is an injective homomorphism $E: \mathbb{F} \rightarrow \hat{\mathbb{P}}$ such that

$$
E\left(x_{i}\right)=1+X_{i}+\text { terms of order } \geq 2 .
$$

Let $\mathbb{P}^{(s)}=\hat{\mathbb{P}} / F_{s+1} \hat{\mathbb{P}}$. The automorphism $\phi_{\sigma}^{(s)}$ induced by a string link $\sigma$ can be converted into an automorphism

$$
\Phi_{\sigma}^{(s)}: \mathbb{P}^{(s)} \longrightarrow \mathbb{P}^{(s)}
$$

using the expansion $E$.

Suppose now we have a singular string link $\tau$ with $k \geq s$ double points. Let $\sigma_{j}, j=1,2, \ldots, 2^{k}$ be the $2^{k}$ string links obtained by resolving the $k$ double points on $\tau$ into positive or negative crossings. Let $\epsilon_{j}=1$ or -1 depending on whether $\sigma_{j}$ has even or odd number of negative resolutions. Then, we say that the string link invariant $\Phi_{\sigma}^{(s)}$ is of finite type in the following sense.

Theorem 2.3. (Lin [?]) In $\operatorname{End}\left(\mathbb{P}^{(s)}\right)$, we have

$$
\sum_{j=1}^{2^{k}} \epsilon_{j} \Phi_{\sigma_{j}}^{(s)}=0
$$

\section{Campbell-Hausdorff invariants are of finite type}

We now use the Campbell-Hausdorff expansion $E: \mathbb{F} \rightarrow \hat{\mathbb{P}}$ given by

$$
E_{C H}\left(x_{i}\right)=\exp \left(X_{i}\right)=\sum_{s=0}^{\infty} \frac{1}{s !} X_{i}^{s} .
$$

For a string link $\sigma$, the induced ring automorphism $\Phi_{\sigma}^{(s)}$ via $E_{C H}$ is completely determined by $\Phi_{\sigma}^{(s)}\left(X_{i}\right), 1 \leq i \leq n$, which are Lie elements in $\mathbb{P}^{(s)}$. We may relate these Lie elements with $\left[\rho\left(l_{i}^{(s)}\right), x_{i}\right], 1 \leq i \leq n$ in the following way.

We have $(3.1) \Phi_{\sigma}^{(s)}\left(X_{i}\right) \equiv \log \left[E_{C H}\left(l_{i}^{(s)}\right) E_{C H}\left(x_{i}\right) E_{C H}\left(\left(l_{i}^{(s)}\right)^{-1}\right)\right] \quad \bmod F_{s+1} \hat{\mathbb{P}}$.

Obviously,

$$
\log E_{C H}\left(\left(l_{i}^{(s)}\right)^{ \pm 1}\right)= \pm \rho\left(l_{i}^{(s)}\right)
$$

with each $x_{j}$ in the right-hand side being replaced by $X_{j}$. 
From now on, we will abuse the notation by identifying these two Lie elements. In general, we will not distinguish Lie elements in $x_{j}$ 's or in $X_{j}$ 's.

Lemma 3.1. With the same notation as in Theorem 2.3, let $l_{i}^{(s)}\left(\sigma_{i}\right)$ be the $i$-th longitude of the string link $\sigma_{j}$. Then

$$
\sum_{j=1}^{2^{k}} \epsilon_{j}\left[\rho\left(l_{i}^{(s)}\left(\sigma_{j}\right)\right), X_{i}\right] \equiv 0 \quad \bmod F_{s+1} \hat{\mathbb{L}}_{X} .
$$

Proof. We argue first inductively that there is a basis for $\hat{\mathbb{L}}_{X} / F_{s+1} \hat{\mathbb{L}}_{X}$, such that for a given base element $e$, its coefficient in $\rho\left(l_{i}^{(s)}\right)$ can be expressed as a polynomial of coefficients of base elements or degree $\leq$ degree $(e)+1$ in $\Phi_{\sigma}^{(s)}\left(X_{i}\right)$.

Suppose we have chosen a basis for $\hat{\mathbb{L}}_{X} / F_{s+1} \hat{\mathbb{L}}_{X}$ up to degree $r+1$ such that for a given base element $e$ of degree $\leq r$, its coefficient in $\rho\left(l_{i}^{(s)}\right)$ is a polynomial of coefficients of base elements or degree $\leq$ degree $(e)+1$ in $\Phi_{\sigma}^{(s)}\left(X_{i}\right)$. We want to extend this basis to a basis up to degree $r+2$ and determine the coefficients of base elements of degree $r+1$ in $\rho\left(l_{i}^{(s)}\right)$.

Notice the following two things:

(1) for $e \in \hat{\mathbb{L}}_{X},\left[e, X_{i}\right]=0$ iff $e$ is a multiple of $X_{i}$; and

(2) by the choice of longitude, $\rho\left(l_{i}^{(s)}\right)$ contains no $X_{i}$.

By 1., we may pick up degree $r+1$ base elements $e$ 's not equal to $X_{i}$ and to extend $\left[e, X_{i}\right]$ 's to a basis of degree $r+2$. By 2., the coefficients of base elements of degree $r+1$ in $\rho\left(l_{i}^{(s)}\right)$ are all lifted up to become coefficients of base elements of degree $r+2$. Then we may use (3.1), (3.2) and the Campbell-Hausdorff formula to finish the induction.

Now we see that there is a basis for $\hat{\mathbb{L}}_{X} / F_{s+1} \hat{\mathbb{L}}_{X}$ such that the coefficient of a given base element in $\left[\rho\left(l_{i}^{(s)}\right), X_{i}\right]$ is a polynomial of coefficients of base elements in $\Phi_{\sigma}^{(s)}\left(X_{i}\right)$. The lemma then follows from Theorem 2.3 and the fact that polynomials of finite type invariants are still of finite type (with compatible order - the maximal number of double points singular links can have so that the invariant does not necessarily vanish on derived alternating sum of links).

We come to our main result of this section now. It says that the Campbell-Hausdorff invariants are of finite type with respect to marked singular links.

Definition 3.1. A marked singular link is a singular link in the usual sense with a marked point on each component away from double points. An 
isotopy of a marked singular link is an usual isotopy with the restriction that the marked points are not allowed to pass through double points.

Let $J$ be a marked singular link with $k$ double points. We may find a disk $D$ in the $S^{3}$ which intersects $J$ transversally at the marked points. This converts $J$ into a singular string link. By picking a base point on $D$ and paths on $D$ connecting the base point and the marked points on $J$, we also get a consistent choice of meridian-longitude pairs for all links $J_{1}, J_{2}, \ldots, J_{2^{k}}$ derived from $J$ by resolving the double points. Such a choice of meridian-longitude pairs is consistent in the sense that if we choose another disk $D^{\prime}$, according to the basic construction of [?], there will be an element $u \in U$ which sends derivations associated with all $J_{j}$ determined by $D$ to that determined by $D^{\prime}$. This will certainly not be true if marked points are allowed to pass through double points.

Thus, we get a well defined invariant of marked singular links

$$
C H^{(s)}(J) \in \mathcal{M}_{s}
$$

which is the image of

$$
\sum_{j=1}^{2^{k}} \epsilon_{j} \partial^{(s)}\left(J_{j}\right) \in \operatorname{Hom}\left(Y, \hat{\mathbb{L}}_{\bar{X}}^{\geq 2}\right)
$$

determined by the consistent meridian-longitude pairs coming from $D$, under the projection

$$
\operatorname{Hom}\left(Y, \hat{\mathbb{L}}_{\bar{X}}^{\geq 2}\right) \longrightarrow \operatorname{Hom}\left(Y, \hat{\mathbb{L}}_{\bar{X}}^{\geq 2} / \hat{\mathbb{L}}_{\bar{X}}^{\geq s+1}\right) \longrightarrow \mathcal{M}_{s} .
$$

Theorem 3.1. Let $J$ be a marked singular link with $k \geq s$ double points. Then

$$
C H^{(s)}(J)=0 .
$$

Proof. We realize $J$ as the closure of a singular string link $\tau$ with $k$ double points. Then $L_{j}$ 's are closures of resolutions of $\tau$. For a fixed consistent choice of longitudes of $L_{j}$ 's, Lemma 3.1 says that the alternating sum of the resulting derivations is zero in $\hat{M} / F_{s} \hat{M}$. Therefore, $C H^{(s)}(J)=0$.

Thus, the invariant of marked singular links derived from $C^{(s)}$ vanishes for every marked singular links with more than $s-1$ double points. It is in this sense we say that the Campbell-Hausdorff invariants are of finite type with respect to marked singular links. See some further discussion at the beginning of Section 5 . 


\section{Quantum invariants can not detect non-invertibility}

A link $L$ is non-invertible if it is not isotopic to the link $L^{\prime}$ obtained by reversing the orientation of each component of $L$. It is a well-known fact that quantum invariants can not detect the non-invertibility of a link. We include here a proof of this fact since we could not find one in the literature. The basic ingredients are all in [?, ?] already and the reader is referred to there for detailed discussions of the construction of quantum invariants.

Let $A=U_{q} \mathfrak{g}$ be the quantized universal enveloping algebra of a simple Lie algebra $\mathfrak{g}$. Together with an invertible element $R \in A \otimes A$ and an invertible central element $v \in A,(A, R, v)$ is a ribbon Hopf algebra. There is also an invertible element $u \in A$ which, among other things, has the following property useful to us.

Let $(V, \rho)$ be an $A$-module and $V^{\vee}$ be its dual. If we identify $V^{\vee \vee}$ with $V$ via the canonical isomorphism, we have

$$
\rho^{\vee \vee}(a)=\rho(u) \rho(a) \rho\left(u^{-1}\right), \quad \forall a \in A .
$$

We will denote by $V^{\lambda}$ the finite dimensional irreducible representation of $U_{h} \mathfrak{g}$ with the highest weight $\lambda$. The key fact we need is that there is an A-linear isomorphism

$$
w_{\lambda}:\left(V^{\lambda}\right)^{\vee} \longrightarrow V^{\lambda^{*}}
$$

such that $\left(\lambda^{*}\right)^{*}=\lambda$ and

$$
w_{\lambda}^{\vee} \cdot\left(w_{\lambda^{*}}\right)^{-1}: V^{\lambda} \longrightarrow\left(V^{\lambda}\right)^{\vee \vee}=V^{\lambda}
$$

is the multiplication by $u v^{-1}$.

Now let $B$ be a braid with $m$ strands. Suppose that all the strands of $B$ point downward. We denote by $B^{-}$the braid obtained from $B$ by reversing the arrow on each strand of $B$. Then we color each strand of $B$ by an $A$-module $V^{\lambda}$, in such a way that the functor $F$ defined in Theorem 5.1 of [?] applied to the colored braid $B_{\lambda_{1}, \ldots, \lambda_{m}}$ gives an automorphism

$$
F\left(B_{\lambda_{1}, \ldots, \lambda_{m}}\right): V^{\lambda_{1}} \otimes \cdots \otimes V^{\lambda_{m}} \longrightarrow V^{\lambda_{1}} \otimes \cdots \otimes V^{\lambda_{m}} .
$$

By Lemma 5.1 of [?], for

$$
F\left(B_{\lambda_{1}^{*}, \ldots, \lambda_{m}^{*}}^{-}\right):\left(V^{\lambda_{1}^{*}}\right)^{\vee} \otimes \cdots\left(V^{\lambda_{m}^{*}}\right)^{\vee} \longrightarrow\left(V^{\lambda_{1}^{*}}\right)^{\vee} \otimes \cdots\left(V^{\lambda_{m}^{*}}\right)^{\vee},
$$

we have

$$
F\left(B_{\lambda_{1}^{*}, \ldots, \lambda_{m}^{*}}^{-}\right)=w_{\lambda_{1}^{*}}^{-1} \otimes \cdots w_{\lambda_{m}^{*}}^{-1} \cdot F\left(B_{\lambda_{1}, \ldots, \lambda_{m}}\right) \cdot w_{\lambda_{1}^{*}} \otimes \cdots \otimes w_{\lambda_{m}^{*}} .
$$

Conjugate further by $w_{\lambda_{1}}^{\vee} \otimes \cdots \otimes w_{\lambda_{m}}^{\vee}$, we get

$$
F\left(B_{\lambda_{1}^{\vee}, \ldots, \lambda_{m}^{\vee}}^{-}\right)=u v^{-1} \otimes \cdots \otimes u v^{-1} \cdot F\left(B_{\lambda_{1}, \ldots, \lambda_{m}}\right) \cdot v u^{-1} \otimes \cdots \otimes v u^{-1},
$$


where

$$
F\left(B_{\lambda_{1}^{\vee}, \ldots, \lambda_{m}^{\vee}}^{-}\right)=F\left(B_{\lambda_{1}, \ldots, \lambda_{m}}^{-}\right)^{\vee}
$$

Now let $\operatorname{Tr}_{q}$ be the quantum trace, i.e.

$$
\operatorname{Tr}_{q}(a)=\operatorname{Tr}\left(u v^{-1} a\right), \quad \forall a \in A
$$

where $\operatorname{Tr}(\cdot)$ is the ordinary trace. Then, we have

$$
\begin{aligned}
\operatorname{Tr}_{q}\left(F\left(B_{\lambda_{1}, \ldots, \lambda_{m}}\right)\right) & =\operatorname{Tr}_{q}\left(F\left(B_{\lambda_{1}, \ldots, \lambda_{m}}^{-}\right)^{\vee}\right) \\
& =\operatorname{Tr}\left(\left(u v^{-1}\right)^{\vee} F\left(B_{\lambda_{1}, \ldots, \lambda_{m}}^{-}\right)^{\vee}\right) \\
& =\operatorname{Tr}\left(u v^{-1} F\left(B_{\lambda_{1}, \ldots, \lambda_{m}}^{-}\right)\right) \\
& =\operatorname{Tr}_{q}\left(F\left(B_{\lambda_{1}, \ldots, \lambda_{m}}^{-}\right)\right) .
\end{aligned}
$$

The value of the quantum invariant on the colored link obtained as the closure of $B_{\lambda_{1}, \ldots, \lambda_{m}}$ is simply $\operatorname{Tr}_{q}\left(F\left(B_{\lambda_{1}, \ldots, \lambda_{m}}\right)\right)$ together with a normalization factor independent of the overall orientation. Therefore, such a quantum invariant can not detect the non-invertibility of a link since it has the same value on the closures of $B_{\lambda_{1}, \ldots, \lambda_{m}}$ and $B_{\lambda_{1}, \ldots, \lambda_{m}}^{-}$.

\section{An example and some comments}

Since the action of $U$ on $\hat{M} / F_{s} \hat{M}$ is unipotent, its restriction on $F_{s-1} \hat{M} / F_{s} \hat{M}$ is trivial. Thus, just like in the case of $\bar{\mu}$-invariant, there is no indeterminacy for the Campbell-Hausdorff invariants when

$$
\rho\left(l_{i}^{(s)}\right) \in \hat{\mathbb{L}}_{\bar{X}}^{\geq s-1}, \quad 1 \leq i \leq n .
$$

In other words, we have an injection

$$
\operatorname{Hom}\left(Y, \hat{\mathbb{L}}_{\bar{X}}^{\geq s} / \hat{\mathbb{L}}_{\bar{X}}^{\geq s+1}\right) \hookrightarrow \mathcal{M}_{s} .
$$

Actually, when $J$ is a marked singular link with $s-1$ double points, it is easy to see that

$$
C H^{(s)}(J) \in \operatorname{Hom}\left(Y, \hat{\mathbb{L}}_{\bar{X}}^{\geq s} / \hat{\mathbb{L}}_{\bar{X}}^{\geq s+1}\right) .
$$

According to Theorem 3.1, this gives us a weight system on marked chord diagrams with $s-1$ chords. Here a marked chord diagram is a usual chord diagram on oriented circles with an additional mark on each circle away from the end points of the chords. Compare with the conventional theory of finite type invariants [?], it is interesting to see that such a weight system can be integrated into a link invariant not living in a vector space. It is certainly desirable to explore such a phenomenon more systematically.

Like $\bar{\mu}$-invariants again, (5.1) makes the computation of non-trivial Campbell-Hausdorff invariants of lowest order quite easy once we have 
the presentation (2.2). In fact, at this level, the Campbell-Hausdorff invariants are exactly the same as $\bar{\mu}$-invariants. So we may use the existing computation of $\bar{\mu}$-invariants in the literature (see [?], for example).

Let us now try to understand how the longitudes change when we reverse the orientation of a link.

Lemma 5.1. Let $L^{\prime}$ be obtained from $L$ by reversing the orientation. We may have a system of longitudes $l_{i}^{(s)} \in \mathbb{F}, 1 \leq i \leq n$, for $L$ such that $\bar{l}_{i}^{(s)} \in \mathbb{F}, 1 \leq i \leq n$, which are obtained by reading $l_{i}^{(s)}$ backward, $1 \leq i \leq n$ respectively, as a word in $x_{1}, \ldots, x_{n}$, form a system of longitudes for $L^{\prime}$.

Proof. We may use the Wirtinger presentation of the link group to get longitudes $l_{i}^{(s)} \in \mathbb{F}$. See [?]. Simply note that when we change $L$ to $L^{\prime}$ by reverse the orientation, a positive (negative) crossing in a projection of $L$ remains to be positive (negative) in $L^{\prime}$. Therefore, when we read the geometric longitudes of $L$ backward as words in Wirtinger generators, we get the geometric longitudes of $L^{\prime}$. Now the process of reducing geometric longitudes of $L$ to $l_{i}^{(s)}$ can also be read backward to get the reduction of geometric longitudes of $L^{\prime}$ to $\bar{l}_{i}^{(s)}$. This finishes the proof.

Example 5.1. Let $L$ be the Borromean rings with appropriate orientation and numbering of components such that

$$
\bar{\mu}(123)=\bar{\mu}(231)=\bar{\mu}(312)=-\bar{\mu}(213)=-\bar{\mu}(321)=-\bar{\mu}(132)=1 .
$$

This implies $C H^{(3)}(L)=-C H^{(3)}\left(L^{\prime}\right) \neq 0 \in \mathcal{M}_{3}$, or

$$
C H^{(3)}(L) \neq C H^{(3)}\left(L^{\prime}\right) \text {. }
$$

Thus, the link invariant $C H^{(3)}$ can detect the non-invertibility of a link and it can not be determined by quantum invariants.

The non-invertibility of a knot is much subtler to detect. The first non-invertible knot was detected by Trotter in 1963 [?]. It is also known that there are knots non-invertible up to concordance [?]. More recently, Kuperberg [?] showed that the Conway's 11-crossing knot is non-invertible by counting epimorphisms of the knot group of Conway's knot into the sporadic simple group $M_{11}$ of order 7920 . Hyperbolic structures on knot complements may also be used to detect non-invertibility of knots [?].

For a knot $K$ with the group $G=\pi_{1}\left(S^{3} \backslash K\right)$, simple homological reasons (Stallings' theorem [?]) imply

$$
\Gamma_{2} G=\Gamma_{3} G=\cdots=\Gamma_{s} G=\cdots .
$$

In particular, the longitude of a knot can not be seen in the quotients of $G$ by its lower central series. For that reason and the nature of Vassiliev knot invariants as well as the current work, we feel more convinced now 
of the truth of the following conjecture which we made several years ago when people started to talk about Vassiliev invariants.

Conjecture 5.1. Vassiliev knot invariants cannot detect the non-invertibility of a knot.

\section{References}

1. D. Bar-Natan, On the Vassiliev knot invariants, Topology 34 (1995) 423-472.

2. __ Vassiliev homotopy string link invariants, J. of Knot Theory and its Ramifications 4 (1995) 13-32.

3. B. Berceanu and S. Papadima, Moduli spaces for generic low-dimensional complexes, J. of Pure and Applied Algebra 95 (1994) 1-25.

4. J. Birman, New points of view in knot theory, Bull. Amer. Math. Soc. 28 (1993) $253-246$.

5. J. Birman and X.-S. Lin, Knot polynomials and Vassiliev's invariants, Invent. Math. 111 (1993) 225-270.

6. T. Cochran, Derivatives of links: Milnor's concordance invariants and Massey's products, Mem. Amer. Math. Soc. 84 (1990) No. 427.

7. N. Habegger and X.-S. Lin, The classification of links up to link-homotopy, J. Amer. Math. Soc. 3 (1990) 389-419.

8. A. Kawauchi, The invertibility problem on amphicheiral excellent knots, Proc. Japan Acad. Ser. A Math. Sci. 55 (1979) 399-402.

9. J. Levine, An approach to homotopy classification of links, Trans. Amer. Math. Soc. 306 (1988) 361-387.

10. X.-S. Lin, Power series expansions and invariants of links, Proc. of 1993 Georgia International Topology Conference, to appear.

11. C. Livingston, Knots which are not concordant to their reverses, Quart. J. Math. Oxford Ser. (2) 34 (1983) 323-328.

12. G. Kuperberg, Detecting knot invertibility, J. of Knot Theory and its Ramifications, to appear.

13. M. Markl and S. Papadima, Moduli spaces for fundamental groups and link invariants derived from the lower central series, Manuscripta Math. 81 (1993) 225-242.

14. J. Milnor, Link groups, Ann. of Math. 59 (1954) 177-195.

15. Isotopy of links, Algebraic Geometry and Topology, Princeton Univ. Press, Princeton 1957.

16. S. Papadima, Campbell-Hausdorff invariants of links, J. London Math. Soc., to appear.

17. N. Yu. Reshetikhin and V. G. Turaev, Ribbon graphs and their invariants derived from quantum groups, Comm. in Math. Physics 127 (1990) 1-26.

18. __ Invariants of 3-manifolds via link polynomials and quantum groups, Invent. Math. 103 (1991) 547-597.

19. J.-P. Serre, Lie Algebras and Lie Groups, Benjamin, New York 1965.

20. J. Stallings, Homology and central series of groups, J. of Algebra 20 (1965) 170-181.

21. H. Trotter, Non-invertible knots exist, Topology 2 (1963) 275-280.

Department of Mathematics, University of California, Riverside, CA 92521

E-mail address: xl@math.ucr.edu 